\title{
CONTRIBUCIONES TEÓRICAS FEMINISTAS A LA COMPRENSIÓN DE LA DIVISIÓN SEXUAL DEL TRABAJO. DE LOS FEMINISMOS MARXISTAS Y MATERIALISTAS A LOS FEMINISMOS DECOLONIALES
}

\author{
Diana Paola Garcés Amaya* \\ Universidad de Granada
}

\section{RESUMEN}

En el presente texto se lleva a cabo un recorrido teórico sobre los aportes de las feministas marxistas, materialistas, decoloniales para comprender la categoría de división sexual del trabajo. Se examinan conceptos relevantes que permiten complejizar el análisis teórico, entre ellos las relaciones sociales de sexo, modo de producción doméstico y consustancialidad de opresiones, comprendiendo cómo las relaciones de género tienen una base material e histórica al mismo tiempo que son estructurantes de la realidad social y que complejizan la experiencia de las sujetas racializadas. Finalmente, se propone retomar la categoría división sexual del trabajo como útil para el análisis en el marco de la globalización neoliberal y como categoría política para visibilizar las condiciones materiales y simbólicas de las mujeres del Sur global. Palabras clave: división sexual del trabajo, feminismo materialista, modo de producción doméstico, relaciones sociales de sexo, consustancialidad de opresiones.

\section{THEORERICAL FEMINIST CONTRIBUTIONS TO THE UNDERSTANDING OF THE SEXUAL DIVISION OF LABOR. FROM THE MARXIST AND MATERIALIST FEMINISMS TO THE DECOLONIAL FEMINISMS}

\section{Abstract}

In this text a theoretical exploration of the Marxist, materialist and decolonial and feminists' contributions is carried out to understand the sexual division of labor category. Relevant concepts that permit a complex analysis such as socio-sexual relationship, domestic production mode and consubstantiality of oppressions are examined, understanding how gender relations have a material and historical base at the same time as are structuring the social reality and making the experience of female racialized subjects be more intricate. Finally, I propose to examine the sexual division of labor as an analysis category in the context of the neoliberal globalization and as a political category to make visible the material and symbolic conditions of women from the global south.

KEYwORDS: sexual division of labor, materialist feminism, domestic mode of production, socio-sexual relationship, consubstantiality of oppressions. 


\section{INTRODUCCIÓN}

La división sexual del trabajo ha sido un concepto ampliamente abordado por los estudios feministas y de género, representando un cúmulo de diferentes reflexiones sobre contextos históricos al igual que de realidades específicas y situadas; además, y gracias a la interdisciplinariedad de la que se ha nutrido, ha sido posible el descentramiento de los análisis mayoritariamente economicistas y universalistas. Adicionalmente, estos aportes han sido sumamente importantes en términos de la riqueza teórica y explicativa, en la medida en que han dado paso a entender las experiencias más concretas y las posiciones diferenciales que ocupan las mujeres en distintas relaciones de dominación, y, por supuesto, de un concepto teórico, ha pasado a ser una categoría política de movilización social tanto para visibilizar experiencias como para demandar cambios urgentes sobre las condiciones de vida.

El presente artículo tiene por objetivo realizar un recorrido teórico desde diferentes corrientes feministas para comprender las formas de apropiación del trabajo producido por las mujeres y la interrelación de las relaciones sociales de sexo (o de género como plantean algunas) con la formación sociohistórica concreta del sistema moderno/colonial de género en sus expresiones actuales. En definitiva, se pretende producir un cuerpo teórico para comprender las bases materiales de la división sexual del trabajo como proceso social y visibilizar sus consecuencias sobre el cuerpo y la vida de las mujeres racializadas.

Entendiendo que "desde donde miremos importa»", la presente reflexión teórica se guía por los aportes que algunas feministas materialistas, ecofeministas y decoloniales que nos permiten complejizar el análisis a partir de las críticas a la economía política hegemónica, los cuestionamientos al conocimiento moderno colonial y a la epistemología que constituye las ciencias sociales; ello, con el propósito de cuestionar los lugares de poder desde los cuales se habla y se construye el conocimiento sobre «las otredades». Siguiendo la aproximación decolonial propuesta por Grosfoguel, entendemos que «hoy día resulta inexcusable para pensadores del Norte global seguir produciendo teoría crítica sin diálogo, con sordera hacia las epistemologías del sur» (97).

A continuación, se abordan los antecedentes teóricos que dieron paso a la formulación del concepto; igualmente, se tratan los aportes principales de las feministas materialistas francesas, que han sido centrales para comprender las relaciones sociales de sexo. Posteriormente se desarrolla el concepto de división sexual del trabajo entendiéndolo como expresión material de las relaciones sociales de género y se da especial importancia al concepto de modo de producción doméstico; del mismo modo, se aborda la discusión sobre la interseccionalidad o consustancialidad de opresiones; y, finalmente, se traen a colación algunos elementos de análisis sobre la actualización de la división sexual del trabajo en el marco de las relaciones sociales

* E-mail: dpgarcesa@correo.ugr.es.

1 Retomando la reflexión de Amaia Pérez. 
y económicas contemporáneas marcadas por la globalización neoliberal y, en específico, se rescatan lecturas sobre la situación latinoamericana.

\section{ALGUNOS ANTECEDENTES TEÓRICOS SOBRE EL ANÁLISIS DE LA DIVISIÓN SEXUAL DEL TRABAJO: LA PROPUESTA DEL SISTEMA DUAL}

Un repaso histórico puede dar cuenta de la existencia de una amplia producción teórica y militante de los feminismos socialistas dentro de la cual se pueden ubicar, en un primer lugar, los debates del pensamiento marxista sobre la opresión de las mujeres. De manera muy concisa, Goldsmith resume los enunciados generales de la siguiente manera:

1. La opresión de la mujer tiene una base material; se originó en la expansión de la producción de mercancías y el surgimiento de la propiedad privada de los medios de producción.

2. La mujer en la sociedad capitalista como ama de casa es una esclava doméstica cuya función estriba en el servicio personal al esposo y a los hijos. El hombre domina a la mujer en virtud de la dependencia económica de esta.

3. En consecuencia, la emancipación de la mujer no puede realizarse dentro de la sociedad capitalista.

4. El socialismo liberará a la mujer, ya que removerá la propiedad privada, socializará las actividades domésticas del hogar e integrará a la mujer a la producción pública (137).

Sin embargo, las mismas condiciones existentes dieron cuenta de que la opresión de las mujeres persistía en los países socialistas, al interior de los movimientos de izquierda y de liberación nacional. Estos hechos motivaron a una nueva ola de producción de conocimiento en los cuales se abordó tanto el análisis de la opresión de las mujeres en la sociedad capitalista como su situación desigual frente al trabajo doméstico. Los nuevos temas del debate intelectual y político motivaron al mismo tiempo la creación de un movimiento autónomo que diera cuenta de las especificidades de la situación de las mujeres, tal y como más adelante lo sería el feminismo marxista y socialista.

En las décadas de 1960 y 1970, llamó centralmente la atención la comprensión teórica y empírica del trabajo doméstico, elemento que hasta entonces no parecía un tema relevante, así que "comenzaron a cuestionarse la forma en que la posición de la mujer estaba particularmente definida por su papel como ama de casa y madre» (GoldsMith 140). En estos análisis existían elementos comunes tales como el entendimiento de que, con el avance del capitalismo, el trabajo doméstico sufre un proceso de privatización, o el hecho de que el trabajo doméstico no puede ser compatible con el trabajo de producción de mercancías.

Un avance significativo en los análisis de la época está relacionado con que la distinción entre trabajo productivo e improductivo «no radica en la forma concreta 
que adopta el trabajo, sino más bien en su forma social y en las relaciones sociales en que está inmerso» (Goldsmith 153). No obstante, aún no era clara la explicación sobre la subordinación específica de las mujeres más allá de dar cuenta de que aquella era anterior al modo de producción capitalista, e igualmente no existía un análisis más profundo sobre las relaciones sociales que ocurren al interior de la familia.

Por su parte, autoras como Young, Rowbotham, Mitchell, Eisenstein y Hartmann abren una nueva tradición para el feminismo socialista al introducir categorías de análisis centrales que venía construyendo el feminismo radical de la época dentro de las que se destacan producción, reproducción, sexualidad y socialización. Estas autoras proponen una lectura sobre la interacción mutua del capitalismo y el patriarcado como sistemas de dominación, siendo la división sexual del trabajo una de las categorías centrales común a todas ellas, categoría que opera tanto para la reproducción del capitalismo como para mantener la subordinación de las mujeres.

En esta corriente se destaca la teoría del sistema dual, en la que, partiendo de superar las limitaciones y los sesgos del marxismo tradicional frente al género, se propone comprender y reconocer la especificidad de las «leyes» del patriarcado, considerando que el capitalismo y el patriarcado serían sistemas de relaciones distintos, pero con un grado de interdependencia considerable. Teóricamente, la apreciación consiste en identificar cómo el proceso de construcción del capitalismo implicó la separación de las esferas productiva y reproductiva. No obstante, hay una tendencia a pretender universalizar la relación que se constituye entre un sistema de producción capitalista y la familia y en este proceso analítico, la familia y la esfera doméstica resultan ser la concreción de las relaciones patriarcales; sin embargo, con esta apreciación se renuncia a formular un análisis de cómo las relaciones entre los géneros tienen una base material incluso en la "esfera productiva».

En este orden de ideas, y siguiendo a Molyneux, se ha criticado tanto una tendencia hacia

el reduccionismo economicista como una recurrencia a los modos funcionalistas de argumentación, al construir la relación entre el capitalismo y el trabajo doméstico, (y finalmente la permanencia de) un enfoque estrecho sobre el trabajo desempeñado en la esfera doméstica, ello a expensas de la teorización del contexto del hogar familiar más amplio (Molyneux 13).

Además de ello, Edholm, Harris y Young, así como Mackintosh, llamaron la atención sobre los análisis ahistóricos y el peligro que se corre al formular conceptos que permiten explicar las relaciones sociales en sociedades capitalistas del Norte global pero que erróneamente pretenden explicar otros contextos sociales y culturales; este era un elemento presente de manera generalizada en los análisis sobre el trabajo de las mujeres en el marco de la familia.

Desde estos avances significativos, se han generado reflexiones para entender que las relaciones de género tienen una base material histórica y que en ese caso pueden considerarse como una división de clase social. A manera de ejemplo Nicholson propone conceder al género un estatus central de análisis, puesto que tiene la capacidad de dar cuenta de los cambios materiales que se dan en el tránsito hacia socie- 
dades de mercado (capitalistas); por lo tanto, es posible considerar al género como una división de clase, "dado que históricamente -las relaciones de género- implican diferente acceso al control de las actividades relativas a la consecución de alimentos y objetos (y de sus recursos y productos)» (42).

Igualmente, Goldsmith llama la atención sobre cómo las labores domésticas no son realizadas homogéneamente por todas las mujeres existiendo relaciones sociales en las que la tecnología, las formas de organización del trabajo, así como la pertenencia a sectores populares y rurales, marcan una diferencia considerable (160).

De manera general, los estudios de género de estos años adelantaron análisis profundos sobre el trabajo doméstico, su definición, las formas en las que este se constituye y se desarrolla, la relación que se establece con el sistema de producción capitalista y sus expresiones en las últimas décadas. Ahora bien, junto a estas propuestas de análisis destacan los objetivos políticos que en ellas subyacen, tales como la revalorización tanto económica como social de los trabajos que realizan las mujeres, la denuncia frente a las limitaciones del concepto tradicional de trabajo (circunscrito a lo productivo), el reconocimiento de la intersección de relaciones de opresión el reforzamiento mutuo del capitalismo y el patriarcado, la visibilidad de la explotación laboral tanto dentro de la familia como en otros espacios de trabajo en el marco, por ejemplo, del descenso del salario y, finalmente, el análisis del papel de las mujeres en las luchas contra los sistemas de opresión y las estrategias para su liberación.

\section{APORTES DE LAS FEMINISTAS MATERIALISTAS FRANCESAS A TRAVÉS DEL CONCEPTO DE «RELACIONES SOCIALES DE SEXO»}

La escuela de pensamiento conocida como feminismo materialista francés ${ }^{2}$ representa una propuesta teórica y política relevante al redefinir e identificar las que serían las bases materiales de la opresión de las mujeres sin obviar las formas de opresión ligadas con las relaciones de clase social, «raza» o etnicidad, que constituyen experiencias diferenciales dentro de la misma clase de sexo.

Rivera resume los aportes de los feminismos materialistas de la siguiente manera:

1) La definición de un modo de producción.

${ }^{2}$ Con feministas materialistas francesas nos referimos al grupo de académicas que ha influenciado la conocida publicación de la revista Questions Féministes, en donde aparecen algunas autoras como Colette Capitan, Christine Delphy, Colette Guillaumin, Emmanuelle de Lesseps, Nicole Claude Mathieu, Monique Plaza, Paola Tabet y Monique Wittig, y que son herederas de los trabajos de De Beauvoir. Así nos guiamos por la propuesta de la publicación La Brecha Lésbica (2005) y de «la combinatoria straight. Raza, clase, sexo y economía política: análisis feministas materialistas y decoloniales» (2017). 
2) La definición de la mujer como clase social y económica.

3) El considerar que las relaciones entre marido y mujer en el seno de la familia son relaciones de producción, y no algo privado, al margen de la historia, o bien una relación sin consecuencias económicas y políticas.

4) El entender que en las relaciones entre hombres y mujeres en general, tanto si pertenecen a la misma clase social como a clases antagónicas, hay en el presente y ha habido a lo largo de la historia un componente importante de tensión, de enfrentamiento, de lucha. Es decir, que las relaciones sociales entre los sexos han sido y son conflictivas (101).

Desarrollando detenidamente esta aproximación, en primera instancia, resulta central situar el hecho mismo de que el «sexo» es una construcción histórica, por lo que «Hombre» $\mathrm{y}$ «Mujer» se constituyen a partir de una relación social antagónica que es material, histórica y concreta. En este sentido, la «clase» es una categoría que pretende ser un instrumento de análisis político, el cual, como lo propone la teoría marxista, da cuenta de la posición que se ocupa en la producción social y frente a los medios de producción.

Lo que deviene de esta afirmación es precisamente que la clase de sexo se refiere a una relación de clase ligada al sistema de producción, a la forma como se organiza el trabajo ("productivo" y «reproductivo») y a la explotación de una clase por otra a través de una «jerarquización naturalizada de los sexos» (SMALdone 5). De manera homóloga entonces a otras clases antagónicas identificadas por la teoría marxista, la clase de los hombres explota a la clase de las mujeres al apropiarse de su trabajo doméstico, reproductivo y productivo, de su cuerpo y de los productos de su cuerpo.

Además del concepto de clase de sexo, autoras como Kergoat desarrollan el término de relaciones sociales de sexo, entendiendo de entrada a la relación social no como un simple vínculo social, sino como una relación: «1) Antagónica, 2) Estructurante para la totalidad del campo social, 3) Transversal a la totalidad de este campo» (33).

En esta propuesta, al entender que las relaciones sociales de sexo son estructurantes de la realidad social, es posible analizar las relaciones entre los sexos al interior del ámbito doméstico y familiar, así como en otros lugares y escenarios del ámbito "productivo», en las unidades de producción familiar y otras formas sociales. Además de ello, da la posibilidad de comprender las transformaciones de dichas relaciones en momentos históricos concretos, su heterogeneidad en diferentes sectores sociales y su base tanto material como simbólica, puesto que «las relaciones sociales de sexo poseen una base material aún cuando tiene también otra (base) de ideas [...] en este caso el naturalismo (o la naturalización de la experiencia de la mujer como esencia) es la ideología de la legitimación» (Kergoat 5).

Dentro de la propuesta feminista materialista francesa (a la que nos hemos venido refiriendo), existe una amplitud de perspectivas que ponen énfasis en las diferentes formas en las que se concretan las relaciones sociales de sexo y en donde se generan los antagonismos entre las clases de sexo.

Siguiendo a Curiel y Falquet, Delphy propone que 
la clase de las mujeres está siendo producida en la relación de explotación del trabajo doméstico de las esposas $-\mathrm{y}$ de los varones menores- por parte de los maridos -y de los hermanos mayores- en el marco de la institución del matrimonio y de la explotación familiar (Curiel Ochy y Falquet Jules 10).

En este orden de ideas, el análisis de la autora toma como punto de partida a la esfera doméstica y la institución de la familia; allí encuentra que estas responsabilidades son exclusivas de las mujeres y que son tareas no remuneradas. Delphy explica la situación de la clase de las mujeres en la medida en que

ella misma (refiriéndose a la mujer como clase) no posee los medios de producción por lo tanto su nivel de vida no depende de las relaciones de producción de clase que pueda mantener con los proletarios, sino de las relaciones de producción de servidumbre que mantiene con su marido [...] mientras el asalariado vende su fuerza de trabajo, la mujer casada la regala; exclusividad y gratuidad están íntimamente ligadas (13).

Esta sería la opresión común a todas las mujeres (incluso en las clases burguesas) según la autora, en la que la división de clases proletario/capitalista no aplica para las mujeres que no trabajan fuera de casa, pero podría pensarse en maridos/ mujeres como clases antagónicas; sin embargo, en este punto vale la pena traer a colación la crítica que Molineux realiza a esta última afirmación:

Claramente, la esposa del burgués, la cual emplea sirvientes para realizar el trabajo del hogar y cuidar de los niños, no comparte la opresión material de las mujeres menos privilegiadas. Esto no resulta cuestionado por el hecho de que sus privilegios pueden derivar de la riqueza del marido o porque ella pueda algún día ser despojada de dichos privilegios por medio del divorcio (30).

Por su parte, Guillaumin desarrolla el concepto de apropiación concreta de la individualidad corporal de la clase de las mujeres explicándolo a partir de la identificación de la relación de poder a la que ella refiere como «imposición y efecto ideológico/discursivo de la idea de naturaleza». De esta manera entiende que la relación social entre los sexos se «soporta no solamente en el acaparamiento de su fuerza de trabajo (la de las mujeres) sino en una relación de apropiación física directa» (GuILLAUMIN 24). Es una apropiación con características similares a las formas de esclavitud, por lo cual Guillaumin ubica en el centro del análisis la existencia de una apropiación y un acaparamiento material del cuerpo, denominándolo como «sexaje», a través de lo que no solo se incluiría a la institución del matrimonio, sino también a las formas colectivas de apropiación en instituciones como la Iglesia, el trabajo o en espacios "públicos», etc., y cuyas expresiones la autora enumera de la siguiente forma:
a) La apropiación del tiempo.
b) La apropiación de los productos del cuerpo.
c) La obligación sexual. 
d) La carga física de los miembros inválidos del grupo (inválidos por la edad -bebés, niños, ancianos- o enfermos y minusválidos), así como los miembros válidos de sexo masculino (26).

Frente a los medios de la apropiación, la misma autora, incluye: a) el mercado de trabajo, b) el confinamiento en el espacio, c) la demostración de fuerza, d) la obligación sexual y e) el arsenal jurídico y el derecho consuetudinario (46). Con ello se logra evidenciar tanto las formas individuales como colectivas y los discursos hegemónicos que naturalizan el lugar subordinado y el trabajo designado arbitrariamente a las mujeres, invisibilizando por completo la existencia misma de la apropiación.

Por otro lado, Wittig propone analizar la ideología de la diferencia sexual como base del régimen de la heterosexualidad, puesto que opera para naturalizar las desigualdades sociales que existen entre hombres y mujeres, es decir, que la diferenciación entre los sexos es la base que justifica la opresión. Con ello da cuenta de que la opresión crea al sexo. Este no es, pues, una realidad a priori ni fuera de lo social, sino que es parte de un discurso político que permea todos los espacios de la cultura y crea realidades.

En tal sentido una sociedad heterosexual impone a las mujeres el rol de reproducir la especie y como consecuencia se identifica que este es un sistema de explotación, ya que este trabajo realizado por la clase de las mujeres es apropiado por la clase de los hombres a través del contrato matrimonial. Este último garantiza esta explotación al hacer que la esposa deje de ser una ciudadana y pase a pertenecer a su marido.

En lo referente al análisis del trabajo doméstico y partiendo de la premisa de D’Argemir de que no existe separación efectiva entre la esfera productiva y reproductiva y que estas no funcionan con autonomía «porque la propia existencia de la producción depende de que, a su vez, tenga lugar el flujo constante de su renovación» (26), es posible identificar que tal división arbitraria de las tareas y actividades nace en un momento histórico concreto y lleva consigo consecuencias sobre la naturalización de los trabajos que realizan las mujeres.

La lectura que realiza Posso afirma lo siguiente:

Dado que el trabajo doméstico se sitúa en la esfera de la reproducción y por fuera de la producción del valor, las categorías del marxismo clásico resultaron insuficientes para estudiar las relaciones económicas y de poder en este ámbito (104).

El modo de producción doméstico es un concepto que puede visibilizar y dar cuenta de las relaciones sociales de sexo en su concreción al interior de la institución de la familia y del contrato matrimonial, aunque deja preguntas sobre las características que tendría en otros escenarios (como el mercado y la "producción»).

Delphy da un salto cualitativo para explicar la especificidad de la explotación del trabajo de las mujeres al conceptualizar el modo de producción doméstico como clave analítica que permitiría entender la situación común de las mujeres en tanto clase de sexo. Además de esto, el «modo de producción doméstico» 
permite entender la situación de las mujeres en otros tipos de relación económica más allá de la condición de asalariadas; por ejemplo, permite entender las condiciones de las mujeres en tanto pertenecientes a la burguesía, al artesanado, o bien al campesinado, partiendo de la premisa de que todas las mujeres realizan trabajo doméstico (en mayor o menor medida dependiendo del consumo, en cuanto a bienes y servicios). Por lo tanto, siguiendo esta línea argumentativa, la especificidad del trabajo doméstico y de la explotación doméstica no se define exclusivamente por la relación de producción en la que se inserte el esposo ni por la venta de la fuerza de trabajo de este.

De otro lado, la autora propone centrar la atención sobre la gratuidad: el trabajo doméstico que realizan las mujeres no tiene valor, ya que estas no reciben un valor de cambio y su trabajo no es intercambiado, lo que da cuenta de que «ni siquiera son propietarias de su trabajo, no lo poseen para intercambiarlo» (DeLPHY 57). Asimismo, esta relación de producción se desarrolla en el marco de una relación de coerción personalizada, puesto que se efectúa para otras personas. De tal manera, es la relación de producción la que explica que estos trabajos se vean excluidos del mundo del valor.

Adicionalmente, como lo menciona Guillaumin:

No sólo se trata de la esposa, sino también de los miembros en general del grupo de las mujeres. Puesto que, en efecto, las madres, hermanas, abuelas, hijas, tías, etc. que no firmaron ningún contrato individual con el esposo, el «jefe de la familia» contribuyen al mantenimiento y a la conservación de los bienes, vivos o no, del mismo (26).

En consecuencia, este análisis materialista de la opresión de las mujeres conduce a identificar a la familia como uno de los espacios en los cuales la explotación económica de las mujeres tiene lugar privilegiado.

\section{LA DIVISIÓN SEXUAL DEL TRABAJO COMO CONCEPTO ESTRATÉGICO}

Ciertamente, el concepto de división sexual del trabajo es el producto de un acumulado de reflexiones, debates y posicionamientos políticos incluso que ha brindado un marco consistente para interpretar las formas específicas y concretas de desigualdad entre los sexos; así, nos referimos a que la categoría de relaciones sociales de sexo resulta tener un nivel de abstracción que no permite aún utilizarla como un marco de referencia para una investigación de las relaciones concretas, sin desmeritar la reflexión profunda y crítica que puedan ofrecer.

En este punto, se trata de concebir la división sexual del trabajo en el plano de las prácticas, como una base observable de lo que serían estas relaciones sociales entre sexo, ya que es allí donde se visibilizan jerarquías, injusticias, segregaciones, etc. Es más, como señala D’Argemir, la ideología de la diferencia sexual construye la desigualdad en la medida en que la división del trabajo expresa la jerarquización 
de tareas, pero también la jerarquización de personas, así como las ideas y representaciones sobre tales actividades y relaciones (18).

Esto último tiene relación con la propuesta teórica multidimensional del género de Scott, para quien «el género es un elemento constitutivo de las relaciones sociales basadas en las diferencias percibidas entre los sexos, y el género comprende cuatro elementos interrelacionados» (23) y en específico, a través de las primeras tres dimensiones interrelacionadas que señala: a) los símbolos y representaciones que se evocan en contextos culturales específicos que pueden ser múltiples y contradictorios, b) la fijación de significados a partir de diferentes instituciones y lugares de saber/poder mediante normas, leyes, doctrinas, etc., es decir, conceptos normativos que definen qué es ser «hombre» y qué es ser «mujer», c) el género se construye a través de la representación/discurso/práctica de forma simultánea a través de diferentes instituciones.

Por lo tanto, el género es un factor estructurante y un elemento que constituye el mundo social, así que no es solo una consecuencia de la división social del trabajo, sino un elemento que lo constituye a partir de esa desigualdad. Las representaciones, atributos y estereotipos de género caracterizan a sujetos y sujetas, orientan la acción y asignan un papel diferencial a hombres y mujeres en ese mundo social en el que se habita.

El trabajo de Benería, por su parte, se concentra en el análisis de la subordinación de las mujeres en el marco de la organización de la producción y reproducción en una sociedad específica. Para ello retoma la conceptualización de la reproducción en su significado amplio, no solo relacionada con la reproducción biológica, sino también con la reproducción social, entendiendo a esta desde cuatro aspectos:

a) Reproducción social (reproducción de las condiciones que sostienen el sistema).

b) Reproducción de la fuerza de trabajo (no solo el mantenimiento cotidiano de trabajadores presentes y futuros).

c) La asignación de agentes a determinadas posiciones en el proceso productivo.

d) La reproducción biológica (Reproducción 65).

Cuando analiza cómo se constituye la organización de la reproducción, hace referencia a la existencia del control de las actividades reproductoras de las mujeres en todas las sociedades: el trabajo del cuidado y el trabajo doméstico ${ }^{3}$. En la medida en que estas actividades se asocian con la reproducción de la fuerza de trabajo se consideran, al mismo tiempo, un dominio fundamentalmente femenino, mientras

${ }^{3}$ Durante el presente texto se hace mención de los dos conceptos «trabajo del cuidado" y «trabajo doméstico» con el propósito de reconocer sus especificidades y no trabajarlos indistintamente o como intercambiables; sin embargo, es claro que dentro de la economía del cuidado se desarrollan diferentes actividades, servicios tendientes a la atención, asistencia por otro/a sujeta, dentro de los cuales el trabajo doméstico adquiere un lugar de suma relevancia. Aunque más que las actividades que se realizan, sus características tienen que ver con la invisibilización y las condiciones desiguales en las que se desarrollan. 
que la participación de las mujeres en el ámbito de la "producción» está condicionada por las responsabilidades en el ámbito de la «reproducción» y la búsqueda de la conciliación de las jornadas laborales.

Esta asignación de trabajos tiene relación con el control de su sexualidad y de su cuerpo y se caracteriza por ser un trabajo gratuito, «invisible», además de que «se considera como "no cualificado" [...] pero, se ignora que en realidad su aprendizaje abarca gran parte del tiempo de socialización de las mujeres» (Poso 103). Así como

ha sido transformado en un atributo natural de nuestra psique y personalidad femenina, una necesidad interna, una aspiración, proveniente supuestamente de las profundidades de nuestro carácter de mujeres. El trabajo doméstico fue transformado en un atributo natural en vez de ser reconocido como trabajo (Federici, Revolución 37).

En su análisis, Benería propone una lectura específica para las relaciones entre producción y reproducción que ocurren en el mundo rural, al mencionar cómo

en las economías rurales hay un alto grado de integración entre el trabajo doméstico y las actividades de la mujer fuera de la casa. A menudo la participación de la mujer en las labores agrícolas y en las actividades mercantiles en una prolongación del trabajo doméstico [...] las muchas horas de trabajo de la mujer rural incluyen no sólo el mantenimiento cotidiano de la familia y las actividades reproductivas, sino también una participación directa en la producción social de valores de uso y de cambio, actividades de circulación, así como trabajo asalariado (Reproducción 58).

No obstante, la misma autora aclara que las actividades que las mujeres realizan en el marco de lo rural varían de acuerdo con las jerarquías de las clases sociales y de la posición social además de las diferentes concepciones simbólicas de cada sociedad sobre el trabajo.

Otra perspectiva de análisis que ofrece la categoría de división sexual del trabajo tiene que ver con el acceso, la posesión o la propiedad de los medios de producción. Nos referimos a los factores productivos como la tierra, el capital, los medios intelectuales y las técnicas producidas por el general intellect. Sin embargo, tanto su acceso como su uso han estado mediados fundamentalmente por el género, y ligados a formas de parentesco, a las prácticas de transmisión de la herencia, así como a las políticas y acciones del Estado.

$\mathrm{Al}$ respecto, los trabajos de Deere, León y Meertens, producidos desde otras latitudes, han dado cuenta de la amplia «brecha de género» existente aún en la propiedad de la tierra en los países latinoamericanos, lo que dificulta las estrategias de las mujeres para generar autonomía, poderes materiales y simbólicos de reconocimiento, así como la posibilidad de tomar decisiones al interior de su familia y su comunidad, y por supuesto de enfrentar las formas de violencias.

Asimismo, Tabet se ha referido a la exclusión de las mujeres de ciertos instrumentos y herramientas que deviene en la exclusión del uso de la fuerza que encierra toda una relación desigual tanto en la participación como en la repartición de los productos. Es decir, que antes de la separación del productor de los medios de 
producción que se da en la transformación de las relaciones de producción feudales a las capitalistas, resulta necesario resaltar que, en un principio, bajo el sistema patriarcal se ha producido una apropiación de los instrumentos de producción, de los instrumentos de la fuerza y de las armas por parte de la clase de los hombres, garantizando la estructura de dominación a través de este monopolio.

Finalmente, dentro de la división sexual del trabajo es necesario llamar la atención sobre rol en la construcción de subjetividades e identidades. D’Argemir reconoce que

los grupos humanos definen su identidad a través del trabajo, porque por muy instrumentales o impersonales que puedan ser las tareas que realicen, para el trabajador se trata de una experiencia personal, de una forma de relacionarse con la realidad en que vive, de identificarse y de ser identificado (34).

En tal sentido, el trabajo no se limita a las actividades que se realizan, sino también al hecho de que se producen y reproducen unas relaciones sociales que tienden a mantener un sistema social específico que incluye la división por sexos, razas, desigualdades geopolíticas, etc. Trabajos feministas como el de Mathieu dan cuenta de que esa relación constitutiva entre la división sexual del trabajo y la naturalización de estas actividades sobre el cuerpo de la clase de las mujeres crea y recrea una identidad femenina.

En otras palabras, las autoras refieren al habitus que, siguiendo a Bourdieu, consiste en la interiorización de una estructura de relaciones de poder que genera prácticas, modos de actuar, autorrepresentarse y percibir el mundo de acuerdo con una posición que se ocupa en determinado espacio social. Esta estructura estructurante opera mediante un proceso de familiarización a través de prácticas sociales que se inscriben dentro de las formas ya fijas del mundo social.

Por lo tanto, el habitus no es simplemente una historia, sino la actuación viva en el presente de todos los aprendizajes a lo largo del pasado; es parte de la reproducción social y de la estructura de clases, en el sentido de que el habitus «es adquirido en una serie de condiciones materiales y sociales, y estas varían en función de la posición en el espacio social» (MARTín 28). Además, el habitus puede generar prácticas de reproducción de la estructura dominante al igual que prácticas de resistencia, dependiendo de la relación entre las condiciones de inculcación y las condiciones en las que opera.

\section{INTERSECCIONALIDAD Y CONSUSTANCIALIDAD COMO APROXIMACIONES TEÓRICAS Y PRÁCTICAS DECOLONIALES}

Además de estas propuestas sobre las expresiones y materializaciones de la explotación y apropiación que tienen lugar en las relaciones sociales entre los géneros, es necesario llamar la atención sobre los grandes aportes realizados por los feminismos negros, latinoamericanos y decoloniales, que denominaré como «feminis- 
mos de color $»^{4}$, como enfoques epistémicos decolonizadores (VIVERos 3), alrededor de los conceptos analíticos de interseccionalidad y consustancialidad de opresiones se hacen visibles las experiencias y las posiciones diferenciales que ocupan las mujeres en distintas relaciones de dominación. Estas propuestas surgieron como denuncia al feminismo «blanco» al estar permeado de prejuicios e ideologías dominantes.

Una de las primeras aproximaciones, que podríamos llamar como clásicas, es la realizada por Crenshaw, quien acunó en 1989 la categoría de interseccionalidad refiriéndose esta «a la forma en que el racismo, el patriarcado, la opresión de clase y otros sistemas discriminatorios que crean desigualdades y estructuran posiciones sociales" $(172)^{5}$, para dar cuenta de las experiencias diferenciales entre hombres/mujeres, puesto que las relaciones de género reproducen formas de «vulnerabilidad», pero también entre mujeres mismas (identificando las relaciones de poder de raza y clase), a fin de comprender con profundidad las formas específicas de discriminación y expandir la protección de los derechos humanos sin caer en enfoques subinclusivos, también ubicando las dificultades en la lectura "objetiva» de la raza o el origen étnico, en la división geopolítica norte/sur, en la reproducción de jerarquías de las élites internas, y los discursos del nacionalismo y la solidaridad racial. Sin embargo, como ella lo aclara, se trata de un concepto de uso práctico sobre los vacíos jurídicos ${ }^{6}$ y no una teoría explicativa de la totalidad de la realidad social.

En específico, la funcionalidad del concepto tiene que ver con la irrupción en el discurso sobre los derechos humanos y la creación de estrategias políticas para, a partir de otra narrativa, entender las injusticias sociales y evidenciar la forma como estas se traslapan para crear múltiples niveles de discriminación y de violencias que contribuyen al desempoderamiento y la subordinación con impactos desproporcionales. Destacando tres niveles en los cuales tiene lugar la interseccionalidad: el estructural, como parte del cruce de sistemas de subordinación; político, que tiene que ver con el policy making y el ordenamiento legal; y el nivel simbólico, que tiene que ver con las representaciones arbitrarias y estereotipadas (LA BARBERA 112) ${ }^{7}$.

4 Retomo este concepto que varias autoras han rescatado del movimiento social, entre ellas, Lugones, quien lo entiende desde su genealogía en el movimiento de mujeres subalternizadas en Estados Unidos y los países del llamado Tercer Mundo y que surgen con una construcción política a lo largo de un proceso de identificación de los antagonismos, mas no como un dato preconcebido sobre rasgos que se naturalizan y escencializan.

5 Traducción propia.

${ }^{6}$ Como en el estudio del caso de la General Motors y la discriminación laboral que experimentaron las mujeres negras en Estados Unidos, el cual daría paso a su propuesta conceptual.

7 La Barbera resume los aportes de la interseccionalidad de la siguiente manera: I) se ubica el foco de atención en el sujeto que se encuentra en el cruce entre distintos sistemas de discriminación, cuya experiencia de discriminación no puede ser explicada usando las categorías de clasificación social de forma aislada; II) se coloca el acento en la simultaneidad de los factores de discriminación; y III) se subrayan los efectos paradójicos de análisis, intervenciones y políticas públicas basadas en un solo eje de discriminación que, abordando separadamente raza, género y clase, crean nuevas dinámicas de desempoderamiento. 
Existe, además de lo anterior, otro elemento que resulta central para dar el salto cualitativo en el entendimiento de las opresiones simultáneas, se trata de «superar la conceptualización aritmética de las desigualdades sociorraciales» (VIVERos 8) y de allí la emergencia de conceptos como el de consustancialidad de las opresiones, o de opresiones simultáneas dando cuenta de la imposibilidad de asilar, jerarquizar y separar las formas de dominación (LugOnes 61).

Así, han sido de marcada importancia aquellas propuestas que enfatizan en llevar a cabo una lectura sobre las experiencias de sexismo racializadas, sobre todo desde los feminismos negros, entre ellas destaca la autora Avtar Brah, quien, desde el lugar de la diáspora en Gran Bretaña, plantea el concepto de «racialización del género" haciendo referencia a la interrelación de diferentes formas de diferenciación social y como parte de procesos históricos específicos de manera tal que va a dar cuenta de los problemas del esencialismo. Igualmente trae a colación el debate sobre la «diferencia» en el feminismo, en donde menciona cómo «nuestro género se construye y representa de distintas formas según nuestra ubicación diferencial dentro de las relaciones globales de poder» (BRAH 131).

De otro lado, el concepto de "matriz de dominación» de Hill Collins, que tiene la intención de dar cuenta de la interacción y prevalencia de procesos tanto macrosociales como microsociales del poder en una sociedad. Ella explica además que

hay dos características en cualquier matriz: a) cada matriz de dominación tiene su particular disposición de sistemas de intersección de la opresión, b) la intersección de sistemas de opresión está específicamente organizada a través de cuatro dominios de poder interrelacionados: estructural, disciplinario, hegemónico e interpersonal. La intersección de vectores de opresión y de privilegio crea variaciones tanto en las formas como en la intensidad en la que las personas experimentan la opresión (130).

Vemos que la explicación de la interconexión y consustancialidad de opresiones debe ser comprendida desde el lugar situado de los sujetos y sujetas, y del funcionamiento concreto de la dominación, las cristalizaciones y las imbricaciones que tienen lugar en la experiencia y en las configuraciones sociales particulares, de la misma manera que Viveros lo expone: «La articulación de las relaciones de clase, género y raza es una articulación concreta, y las lógicas sociales no son iguales a las lógicas políticas» (10).

Sin embargo, el mayor énfasis que realiza Hill Collins se encuentra en rescatar las experiencias de resistencia y la histórica trayectoria de militancia de los movimientos feministas de color. Pensemos en que esta categoría, como lo menciona Viveros, se propone como un paradigma alternativo que busca cuestionar incluso la epistemología occidental (Viveros 6). Además, es en la última década cuando en el contexto académico latinoamericano se empieza a hablar de la interseccionalidad $^{8}$, pero lo cierto es que los procesos colectivos y movimientos de mujeres ya

\footnotetext{
8 Véase, por ejemplo, el trabajo de Viveros Vigoya (2012).
} 
habían llevado este tema a la praxis y a la discusión política? . A partir de allí surgen amplias referencias a la interacción entre las relaciones de género, raza y clase, que tienen implicaciones tanto epistemológicas como políticas.

Ahora bien, en contra de una metanarrativa de la división sexual del trabajo, D’Argemir llama la atención sobre la necesidad, justamente, de desarrollarla dentro de un análisis situado complejo:

Las diferencias de clase, los sistemas de género, las divisiones raciales, son elementos constitutivos del trabajo porque no solo se crean y se reproducen a través de él, sino también lo estructuran y le dan forma. El trabajo se relaciona con distintas formas de desigualdad (13).

Incluso, como lo explica Kergoat:

Una mujer no se piensa como mujer, se piensa también en una red de relaciones sociales: como trabajadora, como joven o vieja, como eventualmente madre o inmigrada. Padece y/o ejerce una dominación según su lugar en esas diversas relaciones sociales. $\mathrm{Y}$ es este conjunto el que va a construir su identidad individual y el que originará sus prácticas privadas (35).

En este sentido, desde una postura feminista materialista se entiende que «toda relación social es sexuada, mientras que las relaciones sociales de sexo se hallan atravesadas por las restantes relaciones sociales» (KergoAT 37), por lo cual no se trata de una lectura interseccional, sino consustancial, evidenciando un entrecruzamiento complejo de las relaciones sociales, así como su interdependencia y reproducción mutua.

En ello coinciden Hill Collins y Davis al enfatizar en la experiencia de las mujeres negras en Estados Unidos, para quienes, al ser situadas no como mujeres sino en la línea de lo no humano a través del proceso de la deshumanización de la esclavización, la intersección de opresiones implica una ubicación específica en la división social y sexual del trabajo, al tiempo que la discriminación racial implica unos significados y unas experiencias muy específicas en el trabajo.

En este orden de ideas, la forma de entender la división sexual del trabajo debe ser crítica frente a los sesgos androcéntricos y etnocéntricos con los que estábamos acercándonos a la realidad, por lo tanto se entiende que el trabajo, las formas en las que se da y el significado dentro del orden simbólico son distintos para cada comunidad humana, de allí que sea útil para entender las complejidades situadas sin dejar de ser un elemento común el hecho de que: a) todas las sociedades reconocen y elaboran diferencias entre los sexos, y b) las actividades masculinas suelen considerarse más importantes que las femeninas (D’Argemir 22).

${ }^{9}$ Véase el legado de la Colectiva del río Combahee, quienes también realizan cuestionamientos a la norma heterosexual, y los trabajos de Francesca Gargallo (2014), en donde se hace un recorrido por los movimientos de mujeres y feministas de América Latina. 
En referencia a lo cual, los feminismos negros también han realizado sus contribuciones:

La división de la esfera pública y privada como aquellos espacios donde se evidenciaba la división sexual del trabajo, perdía ese sentido absoluto cuando se trataba de la experiencia de muchas mujeres en la que ambas esferas fueron espacios de explotación económica, de construcción de estereotipos y roles sexualizados y racializados, ubicadas siempre en esferas laborales menos valoradas y remuneradas, como lo fue, entre otros, el trabajo doméstico (Curiel 5).

Curiel ${ }^{10}$ evidencia que el análisis que el feminismo «blanco» ha realizado sobre la escisión de la esfera pública y privada como base para la reproducción de una división sexual del trabajo y realidad material para las «mujeres» en la medida en que las relega al ámbito doméstico y de la reproducción es una cuestión que escapa al análisis de la realidad concreta de las mujeres afroamericanas. Esto mismo lo evidencia Angela Davis, cuando en su maravilloso texto realiza diferentes relecturas feministas del blues femenino: «Una mujer negra, pobre de la época, que fuera abandonada o rechazada por su amante masculino, no está sufriendo meros problemas privados, sino que está atrapada además en una compleja red de circunstancias históricas» (DAvis, I used to be 157). Con sus letras, el blues femenino detonaba la ideología y las representaciones dominantes de las mujeres, las mujeres negras no tenían la protección del mundo privado.

La diferencia teórico-metodológica por la que apuestan las autoras militantes de los feminismos de color también ha ahondado en comprender las formas en las que la raza, el género y la clase han tenido especiales implicaciones en las colonias latinoamericanas y cómo han persistido ciertas formas de poder colonial que actualmente siguen afectando a las mujeres. En este contexto, el concepto de colonialidad de género propuesto por Lugones tiene el propósito de situar la realidad de «las mujeres de color» en el marco de la cartografía del poder global desplegada a partir de un sistema moderno/colonial de género ${ }^{11}$ y su actualización en el contexto de globalización neoliberal.

Para abordar una lectura aproximativa sobre este escenario, -en sí es contradictorio y complejo- se puede entender que los procesos sociales de largo aliento vienen reorganizando/reestructurando la división del trabajo que modifica, transforma e interviene de manera simultánea en las relaciones sociales de sexo, raza y clase. Siendo claro que el sistema mundo global está constituido por las relaciones de raza, clase, sexualidad y género; en definitiva, y retomando la reflexión Grosfo-

${ }^{10}$ Desde su lugar de enunciación como feminista, negra, lesbiana y latinoamericana.

${ }_{11}$ Es el concepto que podría condensar la propuesta decolonial teórico-política de Lugones. Partiendo de dos marcos analíticos: la interseccionalidad y el análisis propuesto por Quijano sobre el patrón de poder global capitalista, debate el concepto totalizante de «raza» en la exposición de Quijano y su concepto de género que termina reproduciendo la idea biologizante colonial. 
guel, en las zonas del ser y del no ser estas experiencias en cuerpos y sujetos concretos cobran diferencias radicales:

Como en la zona del no-ser, los conflictos de clase, género y sexualidad son, al mismo tiempo, articulados por la opresión racial, los conflictos son gestionados y administrados con métodos violentos y de apropiación continua. La opresión de clase, sexualidad y género vivida por el «no-ser otro» es agravada debido a la articulación de dichas opresiones con la opresión racial [...]. El problema es que la mujer no-occidental y los gays/lesbianas no occidentales en la zona del no-ser son oprimidos no solamente por los pueblos occidentales que habitan en la zona del ser sino también por otros sujetos pertenecientes a la zona del no-ser (101).

Para abordar estos aportes, se recurre a una revisión de la producción teórica e historiográfica de las autoras como Federici, Hartsock, Mies, Ezquerra y Mendoza, quienes retoman las herramientas del materialismo histórico y la aproximación marxista sobre la acumulación originaria para evidenciar la continuidad de estas estructuras y sobre todo identificar las particularidades de las propuestas de la consustancialidad desde el lugar situado de América Latina.

Así, el trabajo de Federici, en primera instancia, analiza el tránsito de un modo de producción feudalista a uno capitalista (europeo) desde la perspectiva de la posición social de las mujeres; allí da cuenta de que el proceso de tránsito se caracteriza por la introducción de una nueva división sexual del trabajo que sometió el trabajo femenino a la reproducción de la fuerza de trabajo mientras que las excluyó del trabajo asalariado al tiempo que producía la mecanización del cuerpo proletario dando origen a la acumulación y reproducción ampliada del capital ${ }^{12}$ y a un nuevo pacto sexual entre varones. nias:

Pero este proceso tuvo características diferentes para las mujeres de las colo-

En las colonias lo vimos con las violaciones masivas de las mujeres indígenas como instrumento de guerra de conquista y asentamiento colonial, la pérdida de estatus social y político, esclavización, reducción a servidumbre, y la intensidad letal del trabajo, entre otras (Mendoza 26),

Además, bajo el proceso de esclavización de las colonias fue posible la emergencia de un ciudadano, paters familia, masculino y blanco en Occidente.

Federici hace énfasis en cómo

las autoridades espańolas introdujeron una nueva jerarquía sexual que privó a las mujeres indígenas de su autonomía y les otorgó a sus parientes de sexo masculino

12 La plusvalía creada por el trabajo asalariado es reinvertida en el proceso de producción (es decir, el plusvalor se convierte en capital). Ver Marx, Karl (2011), «Tomo I, Sección séptima, Capítulo xxII, Acumulación y reproducción ampliada». En El Capital. Biblioteca de autores socialistas. Siglo XXI editores. 
más poder sobre ellas. Bajo las nuevas leyes, las mujeres casadas se convirtieron en propiedad de los hombres y fueron forzadas (contra la costumbre tradicional) a seguir a sus maridos a casa (CALIBÁN 168).

En segunda instancia, las feministas comunitarias y decoloniales, además, proponen el reconocimiento de un patriarcado específico en América Latina en el marco de lo cual es necesario reconocer un patriarcado anterior a la colonia en el territorio de Abya Yala ${ }^{13}$, en donde los varones tendrían un lugar de privilegios dentro de una estructura jerárquica pero dual del género, lo que no es equivalente a la idea binaria del género implantada por el orden colonial.

Esta estructura entra en contacto con el patriarcado moderno-colonial a partir de la intrusión dando paso a la transformación de las relaciones de la aldea en detrimento del poder simbólico y material de las mujeres, Segato ha hecho énfasis en tres factores importantes que dan cuenta de estas transformaciones y entre ellos destaca el papel de privilegios de género que empezaron a ocupar los hombres de la comunidad y su mayor visibilidad en la esfera pública, el confinamiento de las mujeres en el espacio doméstico y privado, y el devenir del género en un estructura dual jerárquica y desigual en términos ontológicos y políticos (31).

Elementos que, a grandes rasgos, constituirían las transformaciones introducidas por el patriarcado colonial moderno y la colonialidad de género, con consecuencias tanto simbólicas como materiales sobre la vida de las mujeres de las colonias.

\section{DIVISIÓN SEXUAL DEL TRABAJO EN EL MARCO DE LA GLOBALIZACIÓN NEOLIBERAL}

Habiendo mencionado la forma en la que se introduce la matriz colonial de género, se pueden encontrar, ahora, diferentes aportes que vinculan claves analíticas más complejas y que ponen en juego el análisis histórico, la imbricación de relaciones de poder (incluyendo la relación de colonialidad) y que realizan un llamado de atención sobre las transformaciones y continuidades de la división sexual del trabajo con relación a una nueva fase de acumulación capitalista en el marco de un sistema mundo global.

Ahora bien, entendiendo el carácter contradictorio del capitalismo, y en especial el contexto de las crisis de los ańos sesenta y setenta, y la tendencia latinoamericana representada en los Planes de Ajuste Estructural y el desmonte del Estado, estudios recientes de Harvey, Estrada y las feministas referenciadas anteriormente, coinciden en que el carácter violento con el que se generó la acumulación primigenia del capital se repite como estrategia de salida a la crisis capitalista, una y otra vez.

${ }_{13}$ Retomamos este término en lengua propia que el pueblo indígena Kuna ha utilizado para nombrar el territorio actualmente conocido como América (término colonial); además, a partir del auge del movimiento indígena en la región ha sido ampliamente movilizado para llevar a cabo su accionar político. Significa en lengua kuna «tierra en plena madurez». 
En este contexto en el que se "actualiza» la acumulación primigenia, la fuerza de trabajo relacionada con el pequeño campesinado y la producción de subsistencia que antes parecía excluida del proceso de acumulación ahora es incluida mediante formas de trabajo de mercado, flexible y precario. Gargallo lo explica de la siguiente manera:

La actual globalización «libera» una gran cantidad de mano de obra del campo, a través de privatizaciones forzadas de la tierra cultivable por las políticas de desarrollo y los planes regionales. Se trata literalmente de explotar las últimas reservas de mano de obra disponibles, muy en particular la femenina y rural, que habían quedado en parte fuera de las relaciones de producción asalariadas (GARGALLO 96).

Además, se plantea que la transformación de las relaciones que intervienen en la reproducción social es estratégica en el marco de asegurar la continuidad de la acumulación; por lo tanto, se trata de «analizar como el sexo, la raza y la clase están siendo movilizados para construir una nueva división social del trabajo a nivel de la familia, de cada país y del conjunto del planeta» (FalQuet, Por las buenas 5).

En los análisis sobre el proceso de restructuración del trabajo en el contexto neoliberal se destaca la perspectiva de housewifisation (Mies, Housewifisation) o feminización del mundo laboral con ella, la intención es destacar cómo las características y las condiciones del trabajo invisible y gratuito de las mujeres empiezan a ser la generalidad en el mundo del trabajo como parte de la acumulación flexible; la flexibilidad, la disponibilidad total, así como la vulnerabilidad, se extienden a diferentes trabajos que no solo desempeñan las mujeres.

No obstante, siendo manifiesto el incremento masivo de la participación de las mujeres en el trabajo remunerado, sobre todo cuando este perdió todas las garantías sociales, ello no implica que sus condiciones sean mejores para las mujeres, sobre todo para las mujeres más empobrecidas del Sur geopolítico.

Menciona Gargallo frente a ello que a las mujeres

se les exige flexibilidad en los horarios y adaptación a actividades diversas, se les despide sin compensación, se controla su fecundidad, se calcula su disposición a trabajar en horarios irregulares, parciales o totales, sin ofrecerles ninguna condición de seguridad para acceder a las maquilas (no es casual que feminicidios y maquilas convivan en todo el territorio mexicano y centroamericano) (95).

Mientras que el desmontaje de las políticas de bienestar social, de los servicios públicos y la privatización de los bienes comunes ha aumentado significativamente el trabajo reproductivo y de cuidado de las mujeres del Sur global.

Frente a ello, las perspectivas de Sassen, Benería, Cobo y Posada proponen las categorías de feminización de la pobreza y de la supervivencia ${ }^{14}$ que pueden dar

14 Aunque este concepto nace desde la década de los setenta, las autoras latinoamericanas lo apropian cada vez más para dar cuenta de las especificidades de las mujeres del Sur global. Ade- 
cuenta del empeoramiento de las condiciones de vida de las mujeres y la creciente vulneración de sus derechos fundamentales. Ello implica que la globalización y la nueva fase de acumulación capitalista han agudizado las cargas de las desigualdades materiales y simbólicas de las mujeres, y asimismo la reproducción del sistema pesa de manera creciente sobre los hombros de ellas: «No sólo los circuitos familiares, sino los gobiernos, las economías nacionales, las empresas que incluso obtienen su tasa de ganancia en la economía ilícita» (SASSEN 43).

Junto a este panorama de pauperización de la vida de las mujeres en Latinoamérica, las autoras Pérez, Sassen, Parella proponen una lectura sobre la crisis de los cuidados producida en el Norte geopolítico, a saber, procesos como el envejecimiento de la población y la reorganización de los roles de género permiten que una mujer del Norte geopolítico que haya ocupado una mayor carga en la jornada de trabajo hasta cierto punto haya adquirido el privilegio de dejar a cargo de otras mujeres el trabajo doméstico y de cuidado de su hogar, de manera que estos trabajos se han relegado, cada vez más, a mujeres que estando indocumentadas o en situación administrativa irregular carecen de derechos de ciudadanía.

Habría que considerar la política cultural en la cual actúa, y cómo los hombres y las instituciones estatales siguen eludiendo la responsabilidad sobre el cuidado tanto en el Norte como en el Sur. De manera que las dificultades de los Estados para garantizar estos servicios a sus habitantes también están enmarcadas en la crisis y el paulatino desmontaje de los Estados de bienestar, pero sumado a ello:

La desigualdad creciente entre países pobres y ricos, la profundización y segmentación de la pobreza en América Latina, la situación descripta de ciudadanía parcial en sus países de origen, la feminización de la pobreza, la segmentación del trabajo por sexo, están motorizando un fenómeno nuevo: la feminización de las migraciones (ZuRUTUZA 5).

Este concepto de la feminización de las migraciones cuestiona los análisis androcéntricos sobre la migración, pero además abre espacios para reflexionar sobre la forma diferencial en la que este fenómeno se experimenta en cuerpos concretos atravesados por el género (además de la etnia, la clase y la ubicación geopolítica), puesto que «los marcos laborales y sociales de inserción de los y las migrantes están estructurados por género" (GUerRa 202). Las mujeres inmigrantes acceden cada vez a trabajos desregularizados (servicio doméstico y prostitución).

El género proyecta una forma de vivir y de experimentar la migración de forma diferencial, «las condiciones laborales se reparten de manera desigual entre los hombres y las mujeres; toda vez que las estrategias de flexibilización y desregulación del mercado de trabajo son un fenómeno claramente sexuado» (PARella 50).

más de ello, como lo explica Arango: «A partir de la década del 80, el debate sobre la "división internacional del trabajo" da paso al de la "globalización", al cual se añaden temas como la transformación de los procesos productivos en las empresas, la introducción de nuevas tecnologías y prácticas gerenciales o el agotamiento del paradigma productivo taylorista/fordista» (30). 
Allí podríamos ver la apropiación del cuerpo de las mujeres, su objetivación para subsumirse a la ley del valor.

En este contexto se señala que la reconfiguración de la división sexual del trabajo no es, pues, un evento pacífico basado en el desarrollo progresivo de la tecnología; por el contrario, más que nunca se reconoce la profundización y generalización de las violencias contra las mujeres y la producción de nuevas formas de violencia. Sobre este asunto, Falquet hace énfasis en los procesos de militarización de territorios y el endurecimiento de las relaciones entre los sexos, mientras Svampa subraya el despliegue de economías extractivistas a lo largo de América Latina y explica cómo allí frente a las responsabilidades del cuidado y la reproducción presenciamos un momento de conflictividad social caracterizado por la amplia participación de las mujeres: «No es que las mujeres no hayan luchado antes, solo que ahora, mucho más que antes, son claramente protagónicas, alimentando nuevas narrativas desde el cuerpo de las mujeres y su relación con la naturaleza» (10).

\section{CONSIDERACIONES FINALES}

A partir el recorrido teórico se puede insistir en que la división sexual del trabajo sigue siendo una categoría útil para comprender cómo se organiza la vida simbólico-material en el marco de relaciones sociales concretas.

Además, la aproximación materialista aporta herramientas teórico-políticas para partir de la explotación y apropiación como características de las condiciones de desigualdad en las que se halla la clase de las mujeres, sin dejar de llamar la atención sobre las experiencias diferenciales de las sujetas que integran esta clase de sexo. Sin embargo, las propuestas permiten entender que, junto con la apropiación de la fuerza de trabajo de las mujeres, se incluye la apropiación física directa, que tiene lugar en la institución del matrimonio y en otras instituciones tales como el trabajo o el Estado. Y por lo demás, estas condiciones de desigualdad se encuentran determinadas por el acceso, la posesión o la propiedad de los medios de producción que tienen lugar.

No obstante, una lectura materialista sobre la división sexual del trabajo debe comprender e incluir un análisis sobre el orden simbólico. Este refiere en primer lugar a los procesos de naturalización de la diferencia sexual (como racial, étnica, etc.) que explican cómo cada sociedad confiere significados diferenciales al trabajo realizado por las mujeres en las esferas de producción y reproducción. Y, en segundo lugar, a la producción de identidades y subjetividades específicas constituidas a partir de prácticas que tienen continuidad en el tiempo (como las que se dan en el marco del trabajo). Con ello, terminan por concluir que, justamente, es la construcción y reproducción de la diferencia la que crea la desigualdad.

Con todo lo seńalado, la corriente materialista se ha nutrido de los grandes aportes realizados por los feminismos negros, latinoamericanos y decoloniales alrededor de la consustancialidad de las relaciones sociales haciendo visibles las experiencias y las posiciones diferentes que ocupan las mujeres en distintas relaciones de dominación, con lo cual el debate se ha complejizado cualitativamente. 
Finalmente, desde una perspectiva de orden estructural, los análisis actuales sobre las reestructuraciones del mundo del trabajo en el marco de «una nueva fase de acumulación capitalista» y la feminización de la supervivencia evidencian que las mujeres siguen siendo las principales responsables de la reproducción y el mantenimiento de la vida.

Recibido: el 11 de febrero de 2019; aceptado: el 23 de septiembre de 2019. 


\section{REFERENCIAS BIBLIOGRÁFICAS}

Arango, Luz Gabriela. «Identidad, género y trabajo en los estudios latinoamericanos». Relations de genre et masculinités en Amérique latine, 39 (2002), pp. 37-58.

Benería, Lourdes. «Reproducción, producción y división sexual del trabajo». Mientras Tanto, 6 (1981), pp. 47-84.

Benería, Lourdes. «Trabajo productivo/reproductivo, pobreza y políticas de conciliación». Nómadas, 24 (2006), pp. 8-21.

Brath, Avtar. Cartografías de la Diáspora. Identidades en Cuestión. Madrid: Traficantes de sueños, 2011.

Сово, Rosa y Posada, Luisa. La feminización de la pobreza. El Periódico Feminista. Mujeres en Red, 2006. Web. Junio 2006.

Сово, Rosa. Hacia una nueva politica sexual. Las mujeres ante la reacción patriarcal. Madrid: La Catarata, 2011.

Colectiva Río Combahee. «Una declaración feminista negra», en Morraga, Cherrie y Castillo, Ana (eds.), Esta puente es mi espalda. Voces de mujeres tercermundistas en los Estados Unidos, San Francisco: Ism Press, 1998, pp. 172-187.

Crenshaw, Kimberlé. "Documento para o encontro de especialistas em aspectos da discriminação racial relativos ao gênero». Estudos Feministas, 171 (2002), pp. 171-188.

Curiel, Ochy y Falquet, Jules (comp.). El patriarcado al desnudo. Tres feministas materialistas. Buenos Aires: Brecha Lésbica, 2005.

Curiel, Ochy. «Los aportes de las de las afrodescendientes a la teoría y la práctica feminista: desuniversalizando el sujeto Mujeres», en Femenías, María Luisa (ed.), Perfiles del Feminismo Iberoamericano, volumen 3, Buenos Aires: Catálogos, 2007, pp. 165-190.

D’Argemir, Dolors. Trabajo, género, cultura. La construcción de desigualdades entre hombres y mujeres. Barcelona: Icaria, 1995.

Davis, Angela. Mujeres, raza y clase. Madrid: Ediciones AkaJ, 2005.

DAVIs, Angela. «I used to be your sweet Mama. Ideología, sexualidad y domesticidad», en Jabardo, Mercedes (ed.), Feminismos Negros, una antología, Madrid: Traficantes de Sueńos, 2012, pp. 135-187.

Deere, Carmen Diana. «Tierra y autonomía económica de la mujer rural: avances y desafíos para la investigación", en Costas, Patricia (ed.), Tierra de Mujeres, Reflexiones sobre el acceso de las mujeres rurales a la tierra en América Latina, La Paz: Fundación Tierra y Coalición Internacional para el Acceso a la Tierra, 2011, pp. 41-64.

Delphy, Christine. Por un feminismo materialista, el enemigo principal y otros textos. Barcelona: la Sal, 1982.

Eisenstein, Zillah. Patriarcado capitalista y feminismo socialista. México D.F: Siglo XXI, 1980.

Estrada, Jairo. Los nuevos paradigmas de la globalización neoliberal y desarrollos contrahegemónicos. Extracto del texto de la ponencia leída en la VI Conferencia Internacional de Estudios Americanos, 2011.

EzQuerra, Sandra. «La crisis o nuevos mecanismos de acumulación por desposesión de la reproducción». Papeles de relaciones ecosociales y cambio global, 24 (2013), pp. 53-62. 
FALquet, Jules. Por las buenas o por las malas. Las mujeres en la globalización. Bogotá: Biblioteca abierta, 2013.

FALQUET, Jules. «La combinatoria straight. Raza, clase, sexo y economía política: análisis feministas materialistas y decoloniales». Descentrada, vol. 1, n. ${ }^{\circ} 1$ (2017), sp.

Federici, Silvia. Calibán y la Bruja; mujeres, cuerpo y acumulación originaria. Madrid: Traficantes de sueños, 2010.

Federici, Silvia. Revolución en punto cero Trabajo doméstico, reproducción y luchas feministas. Madrid: Traficantes de sueños, 2013.

Gargallo, Francesca. Mujer y violencia: el feminismo en la era de la globalización. México D.F.: Universidad Autónoma de la Ciudad de México, 2010.

Gargallo, Francesca. Feminismos desde Abya Yala. Ideas y proposiciones de las mujeres de 607 pueblos en nuestra América. México: Corte y Confección, 2014.

Goldsmith, Mary. «Análisis histórico y contemporáneo del trabajo doméstico», en Rodríguez, Dinah y Cooper, Jennifer (comps.), Antología: Debate sobre el trabajo doméstico, México D.F.: UNAM, 1979, pp. 121-175.

Grosfoguel, Ramón. La descolonización del conocimiento: diálogo crítico entre la visión descolonial de Frantz Fanon y la sociología descolonial de Boaventura de Sousa Santos. Berkely: Berkeley University, 2011.

Guerra Palmero, María José. «Migraciones, Género y Ciudadanía. Una Reflexión Normativa». Astrolabio, 13 (2012), pp. 201-210.

Guillaumin, Colette. «Práctica del poder e idea de Naturaleza», en Curiel, Ochy y Falquet, Jules (eds.), El patriarcado al desnudo. Tres feministas materialistas, Buenos Aires: Brecha Lésbica, 2005, pp. 19-57.

Hartmann, Heidi. El infeliz matrimonio entre marxismo y feminismo: hacia una unión más progresista. Barcelona: Fundación Rafael Campalans, 1991.

Hartsock, Nancy. A new moment of primitive accumulation. Washington: University of Washington press, 2011.

Harvey, David. Los límites del capitalismo y la teoría marxista. México D.F.: Fondo de Cultura Económica, 1990.

Hill Collins, Patricia. Black Feminist Thought. Knowledge, Consciousness, and the Politics of Empowerment. New York: Routledge, 2000.

Kergoat, Daniele. «A propósito de las relaciones sociales de sexo», en Hirata, Kergoat y ZylberBerg, Hocquard (eds.), La División Sexual del Trabajo. Permanencia y cambio, Buenos Aires: Conicet, 1997, pp. 20-50.

La Barbera, Maria Caterina. «Interseccionalidad, un “concepto viajero”: orígenes, desarrollo e implementación en la Unión Europea». Interdisciplina 4, n. ${ }^{\circ} 8$ (2016), pp. 105-122.

León, Magdalena. La desigualdad de género en la propiedad de la tierra en América Latina. Ginebra: IHEID, 2010.

LugONES, M. «Multiculturalismo radical y feminismos de mujeres de color». RIFP, 25, 2005, pp. 61-75.

Martín, Enrique. «Habitus», en Román, Reyes (coord.), Diccionario Crítico de Ciencias Sociales. Terminología Científico-Social, Madrid y México D.F.: Plaza y Valdés, 2009, pp. 25-35. 
Mathieu, Nicole Claude. «¿identidad sexual/sexuada/ de sexo? Tres modos de conceptualización de la relación entre sexo y género", en CUriel, Ochy y Falquet, Jules. (comps.), El patriarcado al desnudo. Tres feministas materialistas, Buenos Aires: Brecha Lésbica, 2005, pp. 130-175.

Mendoza, Breny. «Hacia un pensamiento propio. La epistemología del sur, la colonialidad de género y el feminismo latinoamericano», en Espinosa Miñoso, Yuderkys (coord.), Aproximaciones criticas a las prácticas teórico-políticas del feminismo latinoamericano, Buenos Aires: En la Frontera, 2010, pp. 19-56.

Meertens, Donny. Mujeres campesinas: recuperando la tierra con equidad. Bogotá: Hechos del Callejón, 2008.

Mies, Maria. Patriarchy and accumulation on a world scale-revisited. Oxford: Green Economics Institute, 2007.

Mies, Maria. «Housewifisation-Globalisation-Subsistence-Perspective», en van Der Linden, Marcel and Heinz, Karl (eds.), Beyond Marx Theorising the Global Labour Relations of the Twenty-First Century, Oxford: Brill, 2013, pp. 34-56.

Mitchell, Juliet. Woman's Estate. New York: Vintage Books, 1973.

Molineux, Maxine. «Más allá del trabajo doméstico», en Rodríguez, Dinah y Cooper, Jennifer (comp.), Antología: Debate sobre el trabajo doméstico, México D.F.: UNAM, 1979, pp. 13-53.

Nicholson, Linda. «Feminismo y Marx: integración de parentesco y economía», en BenHabib, Seila y Cornella, Drucilla (ed.), Teoría feminista y teoría crítica, Valencia: Edicions Alfons El Magnánim, 1990, pp. 23-50.

Pérez Orozco, Amaia. «Cadenas Globales de Cuidado». Género, Migración y Desarrollo. Documentos de trabajo 2 (2007), pp. 1-10.

Parella Rubio, Sonia. «Familia transnacional y redefinición de los Roles de Género. El caso de la migración boliviana en España». Papers 97, vol. 3 (2012), pp. 661-684.

Posso, Jeanny. La inserción laboral de las mujeres inmigrantes negras en el servicio doméstico de la ciudad de Cali. Cali: Universidad del Valle, 2008.

Rivera, María-Milagros. Nombrar el mundo en femenino: pensamiento de las mujeres y teoría. Barcelona: Icaria, 1994.

Row вотнам, Sheila. Women, Resistence and Revolution. New York: Pantheon, 1972.

Sassen, Saskia. Contrageografías de la globalización. Género y ciudadanía en los circuitos transfronterizos. Madrid: Traficantes de sueños, 2003.

Scotт, Joan. «El género: Una categoría útil para el análisis histórico», en LAmas, Marta (comp.), El género: la construcción cultural de la diferencia sexual, México: PUEG, 1996, pp. 265-302.

SEgato, Rita. «Género y colonialidad: en busca de claves de lectura y de un vocabulario estratégico descolonial», en Bidaseca, Karina y VÁzquez, Vanesa (comps.), Feminismos y poscolonialidad. Descolonizando el feminismo desde y en América Latina, Buenos Aires: diciones Godot Colección Crítica, 2011, pp. 17-48.

Smaldone, Mariana. Un legado beauvoiriano: El trabajo doméstico desde la perspectiva de Christine Delphy. La Plata: Centro Interdisciplinario de Investigaciones en Género, Universidad Nacional de La Plata, 2013.

Svampa, Maristella. «Megaminería, extractivismo y patriarcado». Presentado en el XXVIII Encuentro Nacional de Mujeres en San Juan, 2013. 
Viveros Vigoya, Mara. «Sexuality and Desire in racialised contexts», en Aggleton, P., Boyce, P., Moore, H. y Parker, R. (eds.), Understanding Global sexualities. New Frontiers, Londres y Nueva York: Routledge, 2012, pp. 218-231.

Viveros Vigoya, Mara. «La interseccionalidad: una aproximación situada a la dominación». Debate Feminista, 52 (2016), pp. 1-17.

Youg, Iris. "Beyond the unhappy marriage: a critique of the dual systems theory", en SARGEnT, Lydia (ed.), Women and revolution, a discussion for the unhappy marriage of marxism and feminism, Boston: South End Press, 1981, pp. 43-69.

Zurutuza, Cristina. Derechos Humanos y Ciudadania Social De Las Mujeres Migrantes: ¿Otro Matrimonio Desavenido? Buenos Aires: CLADEM, 2003. 